\title{
The QCD phase diagram at low baryon density from lattice simulations
}

\section{Owe Philipsen*}

Institut für Theoretische Physik, Goethe-Universität, Max-von-Laue-Str. 1, 60438 Frankfurt am Main, Germany

E-mail: philipseneth.physik.uni-frankfurt.de

The QCD phase diagram as a function of temperature, $T$, and chemical potential for baryon number, $\mu_{B}$, is still unknown today, due to the sign problem, which prohibits direct Monte Carlo simulations for non-vanishing baryon density. Investigations in models sharing chiral symmetry with QCD predict a phase diagram, in which the transition corresponds to a smooth crossover at zero density, but which is strengthened by chemical potential to turn into a first order transition beyond some second order critical point. This contribution reviews the lattice evidence in favour and against the existence of a critical point.

35th International Conference of High Energy Physics - ICHEP2010,

July 22-28, 2010

Paris France

${ }^{*}$ Speaker. 

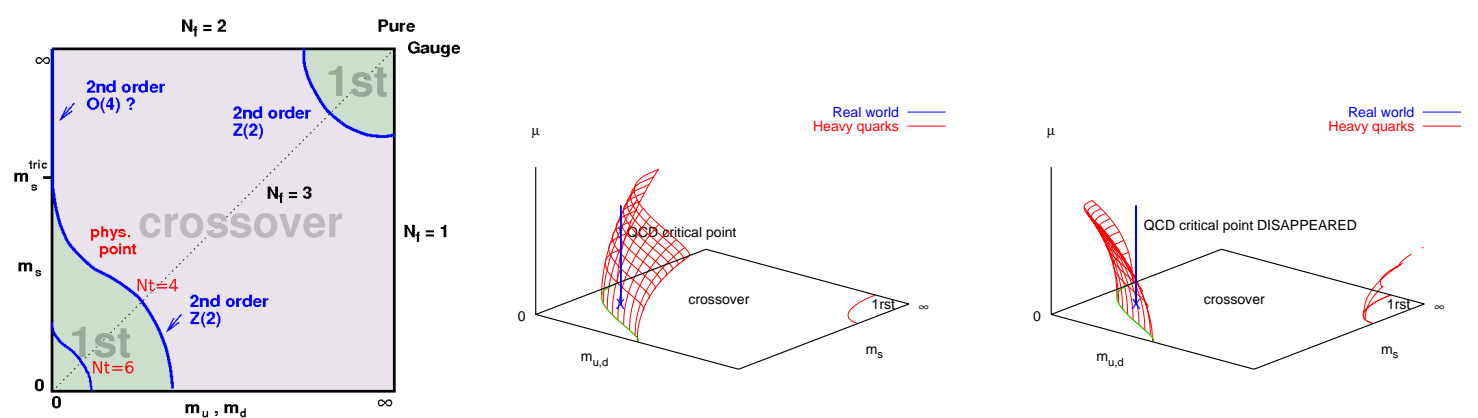

Figure 1: Left: Schematic phase transition behaviour of $N_{f}=2+1$ QCD for different choices of quark masses at $\mu=0$. On finer lattices $\left(N_{t}=6, a \sim 0.2 \mathrm{fm}\right)$, the chiral critical line moves towards smaller quark masses. Middle and Right: Critical surface swept by the chiral critical line as $\mu$ is turned on. Depending on its curvature, a QCD chiral critical point is present or absent.

\section{Introduction}

The QCD phase diagram determines the forms of nuclear matter under different conditions given by temperature, $T$, and chemical potential for baryon number, $\mu_{B}=3 \mu$. Based on asymptotic freedom, one expects at least three different regimes: hadronic (low $\mu_{B}, T$ ), quark gluon plasma (high $T$ ) and colour-superconducting (high $\mu_{B}$, low $T$ ). Whether and where these regions are separated by true phase transitions has to be determined by first principle calculations and experiments. Since QCD is strongly coupled on nuclear scales, Monte Carlo simulations of lattice QCD are the natural approach. Unfortunately, the so-called sign problem prohibits straightforward simulations at finite baryon density. There are ways to circumvent this problem in an approximate way, all of them valid for $\mu / T \lesssim 1$ only [1]: reweighting, Taylor expansions in $\mu / T$ about zero and simulations at imaginary chemical potential, where there is no sign problem, followed by analytic continuation. Within the range $\mu / T \lesssim 1$, all give agreeing results for, e.g., the phase boundary $T_{c}(\mu)$ [2]. Because of the costly finite size scaling analyses, determining the order of the transition, and hence the existence of a chiral critical point, is a much harder task.

The order of the QCD phase transition at zero density depends on the quark masses, Fig. 1 (left). In the limits of zero and infinite masses, order parameters corresponding to the breaking of a global symmetry can be defined, and one finds first order phase transitions at some finite temperatures $T_{c}\left(m_{q}\right)$. On the other hand, one observes analytic crossovers for intermediate masses, separated from the first order regions by second order lines with $Z(2)$ (3d Ising) universality $[3,4,5]$. These are called chiral and deconfinement critical lines, respectively. The former has been mapped out on coarse $N_{t}=4(a \sim 0.3 \mathrm{fm})$ lattices [6] and puts the physical quark mass configuration in the crossover region. The chiral critical line recedes strongly with decreasing lattice spacing [7, 8], i.e. in the continuum the physical point is deeper in the crossover region, Fig. 1 (left).

When a chemical potential is switched on, the chiral critical line sweeps out a surface, as shown in Fig. 1 (right). According to standard expectations [9], for small $m_{u, d}$, the critical line should continuously shift with $\mu$ to larger quark masses until it passes through the physical point at $\mu_{E}$, corresponding to the endpoint of the QCD phase diagram. This is depicted in Fig. 1 (middle), where the critical point is part of the chiral critical surface. However, it is also possible for the chiral critical surface to bend towards smaller quark masses, cf. Fig. 1 (right), in which case there would be no chiral critical point or phase transition at moderate densities. 

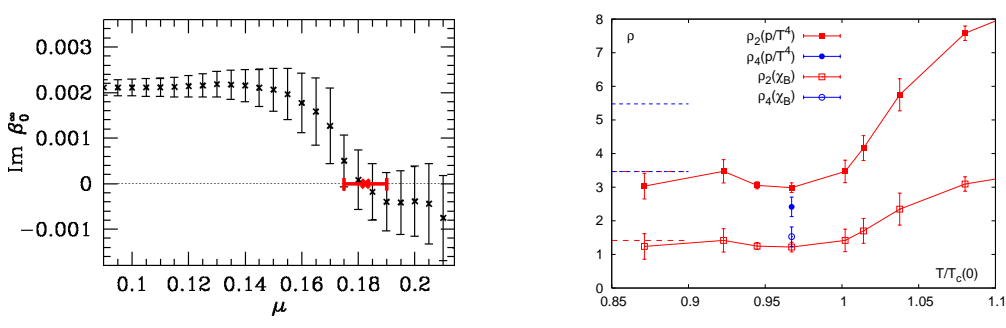

Figure 2: Left: Imaginary part of the Lee-Yang zero closest to the real axis [10]. Right: Estimates for the radius of convergence from different observables and at different order for $N_{f}=2+1, N_{t}=4$. Dashed lines correspond to hadron resonance gas values [13].

\section{Critical point for fixed masses: reweighting and Taylor series}

Reweighting methods at physical quark masses on $N_{t}=4$ lattices get a signal for a critical point at $\mu_{B}^{E} \sim 360 \mathrm{MeV}$ [10]. A Lee-Yang zero analysis was employed, Fig. 2. For a crossover the partition function has zeroes only off the real axis, whereas for a phase transition the zeroes accumulate and pinch the real axis when extrapolated to infinite volume. A caveat of this calculation is the observation that the critical point is found in the immediate neighbourhood of the onset of pion condensation in the phase quenched theory, which is where the sign problem becomes maximal [11]. Therefore, a confirmation with an independent method is desired.

In principle the determination of a critical point is also possible via the Taylor expansion. In this case true phase transitions will be signalled by an emerging non-analyticity, or a finite radius of convergence for the pressure series about $\mu / T=0$, as the volume is increased. The radius of convergence gives the distance between the expansion point and the nearest singularity of the full function, and may be extracted from the high order behaviour of the series. Theorems ensure that if the limit exists and asymptotically all coefficients of the series are positive, then there is a singularity on the real axis. The current best attempt is based on four consecutive coefficients, and a critical endpoint for the $N_{f}=2$ theory was reported in [12]. There are also difficulties in this approach. Firstly, different definitions for the radius of convergence are only unique in the asymptotic limit, but differ at finite order. Estimates at a given order are neither upper nor lower bounds on an actual radius of convergence. Finally, one also obtains finite convergence radius estimates from the Taylor coefficients of the hadron resonance gas model, even though that model does not feature a non-analytic phase transition. This is illustrated in Fig. 2 (middle) [13].

\section{The chiral critical surface}

For definiteness, let us consider three degenerate quarks, represented by the diagonal in the quark mass plane, Fig. 1 (left). The critical quark mass corresponding to the boundary point has an expansion $m_{c}(\mu) / m_{c}(0)=1+c_{1}(\mu / \pi T)^{2}+\ldots$ Tuning to $m_{c}(0)$, one may evaluate the leading coefficients of this expansion. In particular, the sign of $c_{1}$ will tell us which of the scenarios is realised. In order to guard against systematic errors, this derivative has been evaluated in two ways. One is to fit the corresponding Taylor series to data generated at imaginary chemical potential, the other to compute the derivative directly [6,14], with compatible results. One finds on $N_{t}=4$ that $c_{1}=-3.3(3), c_{2}=-47(20)$ [14]. The same behaviour is found for non-degenerate quark masses. 
For physical strange quark mass we calculated $m_{c}^{u, d}(\mu)$ with $c_{1}=-39(8)$ [15]. Hence, on coarse lattices the region of chiral phase transitions shrinks with real chemical potential and there is no chiral critical point for $\mu_{B} \lesssim 500 \mathrm{MeV}$, Fig. 1 (right). Note that one also observes a weakening of the phase transition with $\mu$ in the heavy quark case [5] as well as a weakening of the transition with isospin chemical potential [16].

\section{Conclusions}

In summary, signals from reweighting and radius of convergence estimates are consistent with a critical point, but their systematics does not yet allow a definite conclusion. On the other hand, following the chiral critical surface with controlled systematics tells us the chiral phase transition weakens with moderate $\mu$, but leads us away from the physical point. Possible scenarios are: if we mistrust the systematics of the former methods, we would conclude for either no critical point at all, or a critical point at larger chemical potential $\mu_{B} \gtrsim 600 \mathrm{MeV}$, where current methods break down. If these calculations hold instead, their critical point at moderate densities would not belong to the chiral phase transition, but to physics unrelated to chiral symmetry breaking.

This work is supported by the German BMBF, grant 06MS9150.

\section{References}

[1] O. Philipsen, arXiv:1009.4089 [hep-lat].

[2] P. de Forcrand and S. Kratochvila, PoS LAT2005 (2006) 167 [hep-lat/0509143].

[3] F. Karsch, E. Laermann and C. Schmidt, Phys. Lett. B 520 (2001) 41 [arXiv:hep-lat/0107020].

[4] P. de Forcrand and O. Philipsen, Nucl. Phys. B 673 (2003) 170 [arXiv:hep-lat/0307020].

[5] S. Kim, Ph. de Forcrand, S. Kratochvila and T. Takaishi, PoS LAT2005, (2006) 166 [arXiv:hep-lat/0510069].

[6] P. de Forcrand and O. Philipsen, JHEP 0701 (2007) 077 [hep-lat/0607017].

[7] P. de Forcrand, S. Kim and O. Philipsen, PoS LAT2007 (2007) 178 [arXiv:0711.0262 [hep-lat]].

[8] G. Endrodi et al., PoS LAT2007 (2007) 182 [arXiv:0710.0998 [hep-lat]].

[9] A. M. Halasz et al., Phys. Rev. D 58 (1998) 096007 [arXiv:hep-ph/9804290].

[10] Z. Fodor and S. D. Katz, JHEP 0404 (2004) 050 [arXiv:hep-lat/0402006].

[11] K. Splittorff, arXiv:hep-lat/0505001. J. Han and M. A. Stephanov, Phys. Rev. D 78, 054507 (2008) [arXiv:0805.1939 [hep-lat]].

[12] R. V. Gavai and S. Gupta, Phys. Rev. D 71 (2005) 114014 [arXiv:hep-lat/0412035].

[13] C. Schmidt, PoS C POD2009 (2009) 024 [arXiv:0910.4321 [hep-lat]].

[14] P. de Forcrand and O. Philipsen, JHEP 0811 (2008) 012 [arXiv:0808.1096 [hep-lat]].

[15] J. T. Moscicki et al., Comput. Phys. Commun. 181 (2010) 1715 [arXiv:0911.5682 [Unknown]].

[16] J. B. Kogut and D. K. Sinclair, Phys. Rev. D 77 (2008) 114503 [arXiv:0712.2625 [hep-lat]]. P. de Forcrand et al. , PoS LAT2007 (2007) 237 [arXiv:0711.0023 [hep-lat]].

[17] A. Roberge and N. Weiss, Nucl. Phys. B 275 (1986) 734. 